\title{
Assessing the risk of suicide at triage
}

\author{
Natisha Sands, RPN, RN, PhD*
}

Course Coordinator, Mental Health Nursing, The School of Nursing, The University of Melbourne, 1/723 Swanston Street, Carlton, Victoria 3053, Australia

Received 23 August 2007; accepted 24 August 2007

\section{KEYWORDS}

Suicide risk;

Emergency triage;

Emergency nursing;

Mental health triage

\begin{abstract}
Summary Mental health related presentations to Australian emergency departments are steadily increasing. There is a growing incidence of depression, substance abuse, and other mental illnesses in the Australian population. Mental health problems will contribute $15 \%$ of the total world disease burden by 2020 . Triage nurses are pivotal to the early detection and management of mental health problems.

The rapid assessment of mental health presentations at triage requires skill, knowledge, experience and confidence. One of the more complex aspects of triage is suicide risk assessment. (c) 2007 College of Emergency Nursing Australasia Ltd. Published by Elsevier Ltd. All rights reserved.
\end{abstract}

\section{Background}

Mental health related presentations to Australian emergency departments (EDs) have been steadily increasing over the past 20 years since the integration of mental health care within generalist health. ${ }^{1}$ For many mental health consumers, the ED has become the first point of call in times of personal crisis. ${ }^{1}$ The lack of availability of specialist mental health services outside of business hours, ${ }^{2}$ coupled with the difficulties mental health consumers often experience accessing crisis services, have undoubtedly contributed to the increased rates in ED presentations. ${ }^{2,3}$ Juxtaposed against this is the growing incidence of depression, substance abuse, and other mental illnesses in the Australian population, which has led to an overall increase in consumers seeking mental health care. ${ }^{4}$ Current trends indicate that by 2020 , mental health prob-

\footnotetext{
* Tel.: +61 3 83440776; fax: +61393474172.

E-mail address: nsands@unimelb.edu.au.
}

lems will contribute to $15 \%$ of the total world disease burden. ${ }^{5}$

Triage nurses working at point of entry to the health care system have become pivotal to the early detection and management of a variety of mental health problems. The rapid assessment of mental health presentations is a challenging area of triage practice that requires a combination of skill, knowledge, experience and confidence. ${ }^{6}$ One of the more complex aspects of mental health triage is performing suicide risk assessment. ${ }^{6}$ Identifying when a consumer is at risk of suicide is one of the core tasks in mental health triage, and by employing rigorous risk assessment methodology; triage clinicians can play a significant role in ensuring patient safety. ${ }^{7,8}$

The main aims of triage suicide risk assessment are to determine:

The severity (urgency) and nature of the person's problems.

- The risk of danger to self or others.

Whether a more detailed risk assessment is indicated. ${ }^{7,8}$ 
Table 1 Risk factors for suicide

Previous history of suicide attempts

History of depression, bipolar disorder

Suicidal thoughts

Suicide intent and plan

The means for committing suicide (e.g. access to firearm)

Feelings of hopelessness, shame

Substance use/intoxication

Recent discharge from hospital (4 weeks)

Other mental illnesses

Serious medical illness

Recent death or suicide of a loved one

Male gender

Adapted from Boyce et al. ${ }^{9}$; De Leo et al. ${ }^{10}$; NSW Health. ${ }^{7}$

The following discussion focuses on the processes involved in suicide risk assessment. Unlike the assessment of medical disorders, where careful observation and measurement can be used to reliably determine a diagnosis and rate urgency, suicide risk assessment inherently involves an assessment of human emotions, thinking and behaviours, which vary considerably with individuals depending on their age, culture, and personal circumstance. ${ }^{8}$ The immense diversity of human behaviour and experience precludes accurate, foolproof suicide risk assessment methodologies, however, developing a greater awareness of the risk factors and potential antecedents for suicide increases the likelihood that significant risk factors will be taken into consideration during an assessment. ${ }^{9}$

\section{Signs, symptoms, and antecedents to suicide}

Suicidal thoughts, plans, and self harm attempts are characteristic symptoms of severe depression, and may also be present in people suffering with a range of other mental health problems, such as anxiety disorders, schizophrenia, drug induced psychosis, post natal depression/psychosis, and personality disorder. ${ }^{9,10}$ As well as this, suicidal ideation, or thoughts of suicide, may also be present in people following a significant personal trauma or loss such as the death of a loved one, in those with serious medical disorders, or following the breakdown of a relationship. ${ }^{9,10}$ It is often difficult for the triage clinician to assess the urgency of the presentation in terms of level of risk, and whether or not the person is safe enough to remain in the community, or they require urgent psychiatric assessment and/or hospitalisation. Patients reporting symptoms of depression such as insomnia, sustained low mood, loss of appetite, and feelings of hopelessness, should be routinely assessed for risk of suicide. Those presenting with psychosis, especially command hallucinations (voices directing the patient), should also be routinely screened for suicide risk as command hallucinations are considered a risk factor for suicide. ${ }^{7}$ There is some evidence to suggest that males between the ages of 25 and 30 years are more at risk of suicide than other groups within the population, thus these demographic variables must also be taken into consideration when evaluating the overall level of risk the patient presents with. ${ }^{11}$ Table 1 presents some of the major risk factors for suicide that should be assessed at triage.

\section{The process of suicide risk assessment}

It is immensely important to follow-up on any information the patient or family communicates that causes you to suspect the patient may be suicidal. ${ }^{8-10}$ While it is often very uncomfortable to probe the patient about intimate details of their private life, triage clinicians have a responsibility to be rigorous when assessing the risk of suicide. Some clinicians have expressed concern that asking about suicide may, in fact, prompt the patient to think about harming themself, however, this fear is unfounded; no one can make another person become suicidal, suicide is a complex, multifactorial phenomenon that is unique for each person. ${ }^{7}$

\section{Forming a rapport}

Despite the characteristically brief encounter between the patient and nurse at triage, it is imperative in suicide risk assessment that the nurse attempts to form a rapport with the patient. ${ }^{7}$ Clearly, the patient will feel more comfortable in discussing the intimate details of their life if they feel comfortable with the triage clinician. It is important for the triage clinician to approach the patient in an open-minded, non-judgemental manner. ${ }^{8,9}$

\section{Interview technique}

The specific suicide enquiry necessarily requires the triage clinician to ask highly personal questions of the patient. This is often difficult for a number of reasons, the most obvious being the lack of privacy afforded to patients presenting to triage in a busy, very public ED. Despite the lack of privacy of the $E D$, the triage clinician can make adjustments to their communication style to facilitate a more private, confidential interaction with the patient. ${ }^{9}$ The use of body language such as leaning towards the patient indicates your engagement and active listening; lowering the volume of your voice demonstrates your respect for the patient's private details, and providing gentle encouragement for the patient's efforts to give an account of themself can also help facilitate assessment. ${ }^{9}$

\section{Screening questions}

The guiding principle for structuring a suicide risk assessment is to gradually (and gently) lead the patient through a series of questions that result in specific information about their past, present, and future thinking about suicide. In particular, the focus of the assessment is on the patient's history of self harm, and their current thoughts about suicide including their plans, and level of intent, and the means they have to act on suicidal thoughts..$^{8,9,11,12}$ Table 2 presents examples of suicide risk assessment screening questions that can be use at triage. 
Table 2 Screening questions

Suicide risk assessment screening questions

History taking

1. Has the person felt like this before?

2. Has the person harmed himself or herself before?

3. What were the details and circumstances of the previous attempt/s?

4. Are there similarities in the current circumstances?

5 . Is there a history of suicide of a family member or friend?

Specific suicide enquiry

1. Have things been so bad lately that you have thought you would rather not be here?

2. Have you had any thoughts of harming yourself?

3. Are you thinking of suicide?

4. Have you ever tried to harm yourself?

5. Have you made any current plans?

6. Do you have access to a firearm? Access to other lethal means?

Adapted from NSW Health. ${ }^{12}$

\section{Actions}

Acutely suicidal people need an immediate response to ensure their safety. Immediate response means not letting the patient wait in the waiting room unsupervised, especially if they are unaccompanied. An acutely suicidal patient requires immediate triage, and the assigning of a triage category should reflect this level of urgency. ${ }^{8,12}$ It is imperative for one-to-one supervision and urgent assessment by ED medical/nurse practitioner and/or mental health triage services to be initiated immediately by the triage nurse.$^{8,12}$ Early identification and management of suicide risk at triage is life preserving.

Competing interests: None declared.

Funding: None declared.

\section{References}

1. DHS. Mental health presentations to the emergency department. Melbourne: Victorian Department of Human Services. Available from: http://www.health.vic.gov.au/mentalhealth/ publications/mh-presentations.pdf; 2005 [cited September 1, 2007].

2. Mental Health Service. A new millennium-a new beginning. Mental health in south Australia. Better practice in emergency mental health demand management. Adelaide, South Australia: Mental Health Service, Department of Human Services.
Available from: http://www.dh.sa.gov.au/mental-health-unit /documents/Emergency\%20Demand/D1-

02\%20Better\%20Practice\%20in\%20EMHDM\%20-

\%20Background\%20Paper.pdf; 2002 [cited September 1, 2007].

3. Knott JC. Management of mental health patients in the emergency department. PhD thesis. Melbourne: Department of Surgery, University of Melbourne. Available from: http://eprints.infodiv.unimelb.edu.au/archive/00002656/; 2006 [cited September 1, 2007].

4. NSW Health. The management of people with a co-existing mental health and substance use disorder-discussion paper. Sydney: Centre for Mental Health, NSW Department of Health. Available from: http://www.health.nsw.gov.au/health-publicaffairs/publications/mhsubuse/SDG(D_paper).pdf; 2000 [cited September 1, 2007].

5. Murray CJL, Lopez AD, editors. Global burden of disease: a comprehensive assessment of mortality and disability from diseases, injuries, and risk factors in 1990 and projected to 2020. Cambridge, MA: Harvard University Press; 1996.

6. Sands N. Mental health triage nursing: an Australian perspective. J Psychiatric Mental Health Nurs 2004;11(2):150-155.

7. NSW Health. Framework for suicide risk assessment and management for NSW Health Staff. Sydney: NSW Department of Health. Available from: http://www.health.nsw.gov.au/ pubs/2005/pdf/risk_assessment.pdf; 2004 [cited September 1, 2007].

8. NZGG, Ministry of Health. Best practice evidence-based guideline: the assessment and management of people at risk of suicide. For Emergency Departments and Mental Health Service Acute Assessment Settings. Wellington: New Zealand Guidelines Group and New Zealand Ministry of Health. Available from: http://www.nzgg.org.nz/guidelines/0005/Suicide Guideline.pdf; 2003 [cited September 1, 2007].

9. Boyce P, Carter G, Penrose-Wall J, Wilhelm K, Goldney R. Summary Australian and New Zealand clinical practice guideline for the management of adult deliberate self-harm (2003). Australasian Psychiatry 2003;11(2):150-155.

10. De Leo D, Cerin E, Spathonis K, Burgis S. Lifetime risk of suicide ideation and attempts in an Australian community: Prevalence, suicidal process, and help-seeking behaviour. J Affective Disord 2005;86(2-3):215-224.

11. DoHA. Living Is For Everyone (LIFE): a framework for prevention of suicide and self-harm in Australia: learnings about suicide. Canberra: Commonwealth Department of Health and Aged Care. Available from: http://www.health.gov.au/internet/wcms/Publishing.nsf/ Content/3B17F057A84FB8D2CA25702D001E946F/\$File//rncover .pdf; 2000 [cited September 1, 2007].

12. NSW Health. Suicide risk assessment and management: Emergency Department. Sydney: NSW Department of Health. Available from: http://www.cs.nsw.gov.au/MHealth/documents/ suicide_ED_protocols.pdf; 2004 [cited September 1, 2007]. 